\title{
Protein-Protein Interaction Interface Database Development based on Protein Network
}

\author{
Mr. Kamlesh K. Gautam \\ Assistant Professor, Information Technology, Rajkiya Engineering College, Chandpur, Bijnor, Uttar Pradesh, India
}

\begin{abstract}
Protein-Protein interaction interface database (PPIIDB) is developing on the basis of cellular and biological processes of protein-protein interaction interfaces of protein network. PPIIDB is collection of dataset of PPII of Breast cancer of Homosapience which developed on the basis of various parameters such as such as Accessible Surface Area (ASA), Buried Surface Area (BSA), Binding energy $(\Delta G)$, Cluster Coefficient (CC), Connectivity, Degree of node or Degree of network, Euclidean Distance between interacted proteins, Network Interaction Modularity (Im), size of interface or Voronoi Interface Area (VIA), Significant Connected Component (SCC), Scoring Function, Combine Association Score (CAS), Surface shell (No of Surface Shell Residue, Surface Shell Area),interface face (no. of Interface Residue, Interface Area), Interior core (No of Interior Residue, Interior Area), Resolution, length etc.
\end{abstract}

Keywords: ppiidb, ppiidatabase, ppii, Protein-Protein Interaction, ppiidbpn, Protein Interface.

\section{PPIID INTRODUCTION}

Protein-protein interactions interface (PPII) of protein network database can perform a big role in many biological functions and processes in field of bioinformatics and biotechnology and also in System biology. This Protein-protein Interaction Interface (PPII) database can be enhancing our knowledge and many biological process and functional in biological system. Protein-Protein interactions interfaces (PPII) also regulate many fundamental cellular processes in biological system. There are many databases are related to protein-protein interaction available like HPRD, DIP, and BIND, IntAct, BioGRID, MINT, OPHID, POINT, PDB, STRING, and nterPare [1]. The Protein-Protein Interaction Interface Database (PPIIDB) is developed on the basis of the Protein-Protein Interaction Interface (PPII) of protein network with various parameters such as combine association score (CAS), buried surface area (BSA), Network Interaction Modularity (Im), Accessible surface area (ASA), Binding energy (G), interface face (No of Interface Residue, Interface Area), Cluster coefficient (CC), connectivity, Degree of node or Degree of network, Euclidean Distance between interacted proteins, size of interface or Voronoi Interface Area (VIA), significant connected component (SCC), Scoring Function, surface shell (No of Surface Shell Residue, Surface Shell Area), interior core( No of Interior Residue, Interior Area), Resolution, length etc.

The interaction interface of Protein is basically developed on the structural data of protein network and some biological and physical parameters of protein-protein Interaction Interface complexes. These entire proteinprotein interaction interfaces are basically taken from the breast cancer of the Homosapience. [5]. we can avail all the PPII parameters required data and values at one and single platform with integrated form of data about PPII.
To achieve this purpose in easy way, we have to develop a PPII database [2].

Protein-protein Interaction Interface (PPII) Database, which is containing experimental data of various parameters of Protein-protein Interaction Interface (PPII) along with various method of interaction methodology, interaction technique of proteins and various type of biological protein networks with structural information and experimental conditions of proteins and its network [1].

The basic mission of the Protein-protein Interaction Interface (PPII) database is to provide an integrated platform where inventors, student, and researchers involved in protein-protein interaction interface studies can find all the necessary data available at single place. Protein-protein interactions interface database form the basis for most cellular and biological processes including events intimately linked to human disease such as breast cancer. The defining to the protein-protein interaction interface was described various way and methodology [4].

\section{PPIIDB DEVELOPMENT}

In this project we have used to protein network and interaction of protein to describe the protein and their relationship status between protein-protein interaction interfaces of PPII database. To create a protein-protein interaction interface (PPII) database model and use this PPII database model to provide possible protein interactions interface database. In PPII the Protein A is base protein or master protein also we can say that is main protein interacts with Protein B that is slave protein or interacted protein with master protein is more accurate than simply saying Protein A interact with Protein B is more essential then the simple protein-protein interaction model network. In this database model, we can describe by 
latent variables and protein-protein interactions interface (PPII) by evident variables the interactions between small protein-protein are estimated and then used to predict possible protein-protein interaction interface (PPII) and also developed their protein network [9].

There are several public databases and repositories available an on the Internet ant that is store PPII identified by experimental and practical methods.

\section{ANALYSES OF VARIOUS DATABASES}

We have analysis various Databases, like the PINT, Protorp, HPRD, BioGRID, BIND, IntAct, MIPS, APID, DIP, and MINT these all databases provide the lists of experimental and practical determined interactions of protein ant its protein networks [10].

This databases of Protein-Protein Interaction interface (PPII) of protein network database is the most importance of prediction to filling this gap of database of PPII on single platform and also its appearance to identify the charged residues at the periphery of the protein-protein Interaction interface (PPII) of protein network that work as a big component of long-range interactions for certain type protein-protein complexes [12]. This database project of PPII has been recognized by a number of groups of database of PPII and then developed to this databases of PPII, there are various database is related of PPII such as OPHID and POINT which predict PPI as well as STRING, a database of predicted Protein-Protein associations (direct and indirect PPI) and also developed its networks [11].

A. In PPIIDB of the human pre disposition genes identified to protein (e.g., BRCA1 and BRCA2) are responsible for less than $5 \%$ of all breast cancer cases. We also explain the combined association score and rate of two proteins interactions interface and their networks, this theory known as electrostatic steering.

\section{B. Abbreviations and Acronyms}

PPII Protein-Protein Interaction Interface

PPIIDB Protein-Protein Interaction Interface Database

ASA Accessible surface area

SASA Solvent Accessible surface area

BSA Buried Surface area

CAS Combine Association Score

CC Cluster Coefficient

SCC Significant Connected Component

DON Degree of Node

HPRD Human Protein Reference Database

DIP Database of Interacting proteins

BIND Bimolecular Interaction Network Database

MINT Molecular Interaction database

PPI Protein-Protein Index

\section{Equations}

Pij

(i) Combined association Score $=\log$------ PiPj (ii) Modularity, $M=\frac{\operatorname{Ind}(\mathrm{S})}{-}$
Outd(S)
(iii) Eint $=$ Evdw + Eelec $(\mathrm{kcal} / \mathrm{mol})$

\section{CONCLUSION}

This database is developed for all biological related student and research scholars and scientist for getting relevant data of Protein-Protein network on a single platform with some their some standard parameters, which are already, define in above documents.

- Provide a framework for decomposing the protein interaction interface network into protein network modules of biological database systems.

- The network of Protein-Protein interaction modules provides a plausible way to understanding the interactions between protein-protein interaction interface models with their interface area.

- With the increasing amounts of protein-Protein interaction interface data and information available, our approach will help construct a more complete view of interconnected functional modules of protein-protein interaction network of the whole biological system due to resolution and also show the network images, which are taken form string.

- We can easily retrieve the various types of required data on single platform, which is related to proteinprotein interaction interface with their values.

In this project, we tried to relate to the protein-protein interactions interface of breast cancer and others protein of homosapience in protein network of biological system as well as in system biology with protein-protein Interactions interaction database (PPIIDB) development.

\section{FUTURE WORK}

Protein-protein Interactions interaction database (PPIIDB Interaction database (PPIIDB) is so much reliable and developed according the need of user and scientist and research scholars. In future prospect we can update to this database day by day according data availability because the protein-protein interaction is regular process so data can be dynamic and we cannot stop it so we can change and update day by day. In this database we can also used and developed some advance parameters and features of protein network.

\section{REFERENCES}

[1] Harpaz et al. 1994; Gerstein et al. 1995;Pontius et al. 1996; Nadassy et al. 2001; McConkey et al.2002; Tsai and Gerstein 2002).

[2] Stelzl, U. and Wanker, E.E."The value of high quality proteinprotein interaction networks for systems biology." In (2006) Curr. Opin. Chem. Biol., 10, 551-558.

[3] Interface Surfaces for Protein-Protein Complexes": Yih-En Andrew Ban, Herbert Edelsbrunner and Johannes Rudolph 
[4] "On the role of electrostatic interactions in the design of proteinprotein interfaces."F.B.SHEINERMAN \& B. HONIG."J. Mol. Biol. 318 (2002), 161-177.

[5] Trends in Genetics, 17, 2001."Protein-Protein interaction maps: a lead towards cellular functions". Legrain, J. Wojcik, and J.M..Gauthier.

[6] "Relating Protein-Protein Interaction and Domain-Domain Interaction" COS557 Project Report Wei Dong May 16, 2006.

[7] PIPs:"Human protein-protein interaction prediction database". Mark D. McDowell, Michelle S. Scott and Geoffrey J. Barton* School of Life Sciences Research, College of Life Sciences, University of Dundee, Dow Street, Dundee, DD1 5EH, UK

[8] Scott, M.S.\&Barton, G.J."Probabilistic prediction and ranking of human protein-protein interactions"BMC Bioinformatics (2007), 8 , 239.

[9] Von Mering,C., Jensen,L.J., Kuhn,M., Chaffron,S., Doerks,T., Kruger,B., Snel,B. and Bork,P."STRING7 - Recent developments in the integration and prediction of protein interactions. Nucleic Acids Res.”. (2007), 35, D358-D362.

[10] V.Wolowski, "Computational analysis of protein-protein complexes related to knowledge-based predictions of interaction", (Diploma Thesis, University of Hagen, Germany, 2008).

[11] "Protein-Protein Interaction Prediction" F.Fink, St. Ederer, W. Gronwald

[12] Han KS, Park BK, Kim HG, Hong JS, Park J: HPID:"The Human Protein Interaction Database". Bioinformatics 2004, 20:24662470.

[13] "A protein domain interaction interface database": InterPare.Gong S, Park C, Choi H, Ko J, Jang I, Lee J, Bolser DM, Oh D, Kim DS, Bhak J.National Genome Information Center, KRIBB, Daejeon, Korea. ssgong@kaist.ac.kr

[14] "Fredric Richard and its independently implemented 3-D" by Michael Connolly in 1983 and Tim Richmond in 1984.

[15] "Construction of Candida Albicans Protein Network from Yeast Protein Interactions in Systems Biology": Chung-Yen Lin1,2 Chieh-Hwa Lin1 Chi-Shiang Cho1,Fan-Kai Lin1 Shu-Hwa Chen2 Chao A. Hsiung1

[16] XU, C. J. TSAI, AND R. NUSSINOV. (1997), "Hydrogen Bonds and salt bridges across protein-protein interfaces.Protein Engin." 10999-1012.

[17] SHARP2:"Protein-protein interaction predictions using patch analysis":Yoichi Murakami and Susan Jones*Department ofBiochemistry, School of Life Sciences, John Maynard Smith Building, University of Sussex, Falmer, Brighton. BN1 9QG

[18] Tsai, J. \& Gerstein, M. (2002) "Calculation of protein volumes: sensitivity analysis and parameter database." Bioinformatics 18:985-995.

[19] Rodier, F., Bahadur, R. P., Chakrabarti, P.\& Janin, J. (2005).Hydration of protein-proteininterfaces.Proteins 60:36-45.

[20] ProViz :"Protein Interaction Visualization and Exploration": ToolDavid Auber, Florian Iragne, Bertrand Mathieu, Macha Nikolskiand David Sherman, LaBRI UMR 5800, Universit'e Bordeaux 1, urs de la Lib'eration, Talence Cedex, France.

[21] Nooren, I. M. \& Thornton, J. M. (2003). Diversity of proteinprotein interactions. EMBO J. 22:3486-3492.

[22] Studies of protein-protein interfaces:a statistical analysis of the hydrophobic effect.Tsai,C. J.,Lin, S.L.,Wolfson, H.J.\&Nussinov,R.(1997).Protein Sci 6:53-64.

[23] Robert, C.H. \& Janin, J. (1998). "A soft, mean-field potential derived from crystal contacts for predicting protein-protein. 